COMMENT. The study confirms that cognitive functions are impaired after cerebellar lesions, and particularly right-sided hemisphere lesions. In accordance with the earlier work of Courchesne E and Allen G (Learn Mem 1997;4:1-35), cerebellar damage does not eliminate function but impairs the performance. The cognitive impairments are not explained by a dysmetria of motor performance but rather a "dysmetria of thought" (authors' term).

\title{
COGNITIVE-BEHAVIOR THERAPY AND SERTRALINE FOR OCD
}

The efficacy of cognitive-behavior therapy (CBT) alone and medical management with the selective serotonin reuptake inhibitor sertraline alone, or CBT and sertraline combined, as initial treatment for children and adolescents with obsessive-compulsive disorder (OCD), was evaluated by a randomized controlled trial conducted at Duke, Penn and Brown Universities. A volunteer outpatient sample of 97 patients with OCD, aged 7 through 17 years, and recruited between 1997 and 2002, received treatment or placebo for 12 weeks. Clinical remission was defined as a Children's Yale-Brown OC scale score less than or equal to 10 . Statistically significant benefits were measured with CBT alone $(p=.003)$, sertraline alone $(p=.007)$, and combined treatment $(p=.001)$ compared with placebo. Combined treatment was superior to CBT alone $(p=.008)$ and to sertraline alone $(p=.006)$. The rates of clinical remission were $53.6 \%$ for combined treatment, $39.3 \%$ for CBT alone, $21.4 \%$ for sertraline alone, and $3.6 \%$ for placebo. The remission rate for combined treatment was not significantly different from that for CBT alone $(\mathrm{p}=42)$ but was superior to sertraline alone $(\mathrm{p}=.03)$ and placebo $(\mathrm{p}<.001)$. The remission rates for CBT alone and sertraline alone were not different $(\mathrm{p}=.24)$ from each other but were different from placebo ( $\mathrm{p}-.002)$. Sertraline adverse events occurred in $5 \%$ of patients, 2 times that with placebo, and included decreased appetite, diarrhea, enuresis, motor overactivity and impulsivity, nausea, and stomachache. None was suicidal. Treatment should begin with combination CBT and SSRI (sertraline) or CBT alone. (Pediatric OCD Treatment Study (POTS) Team. Cognitivebehavior therapy, sertraline, and their combination for children and adolescents with obsessive-compulsive disorder. A randomized controlled trial. JAMA October 27, 2004;292:1969-1976). (Respond: John S March MD MPH, Department of Psychiatry, DUMC Box 3527, Durham, NC 27710).

COMMENT. The authors note that OCD affects approximately 1 in 200 young patients and most are initially treated with SSRIs. Duke and Penn University have favored management with CBT (March JS et al. J Am Acad Child Adolesc Psychiatry 1994;33:333-341), and the results of the present study establish the superiority of CBT or CBT combined with SSRI to SSRI alone. A greater availability of CBT should benefit and improve the quality of life of patients with OCD.

Quality of life in children with ADHD. A survey conducted in the ADHD Clinic in British Columbia, Canada, that included 165 respondents, showed that children with ADHD have more parent-reported problems with emotional behavior, mental health, and self-esteem than normal. These effects have an impact on the parents' emotional health and family activities. The adverse effect on health-related quality-of-life (HRQL) correlates with parent-reported inattentive, hyperactive, and combined symptoms of ADHD. Children with more severe 
symptoms of ADHD and comorbidities had poorer psychosocial HRQL. Research and management of ADHD should include measurement of broader domains of family impact and child health. (Klassen AF, Miller A, Fine S. Pediatrics November 2004;114:e541-e547).

Negativity in the mother-infant interaction and early family adversity each contribute to later hyperkinetic symptoms in a study of the effects of regulatory problems in infancy. (Becker K, yet al. Acta Paediatr Nov 2004;93:1463-1469).

\section{SEIZURE DISORDERS}

\section{CONSEQUENCES OF INAPPROPRIATE ER MANAGEMENT OF STATUS EPILEPTICUS}

The clinical features of status epilepticus (SE) requiring admission to pediatric intensive care (PIC) and the emergency pre-PIC treatment of SE compared to standard guidelines were studied in relation to the course of SE after admission to PIC at Great Ormond Street Hospital for Children, London, UK. The records of 98 (4\% of all admissions) children (median age 2.2 years, range 0.1-12.1 years) with confirmed episodes of SE were reviewed. The majority of patients were under 5 years of age. The mean duration of SE was 90 minutes (range 30-435 min) and was independent of etiology. Diazepam or lorazepam was most commonly administered as emergency pre-PIC treatment, but the dose was frequently lower than that recommended. The risk of respiratory insufficiency following seizure termination is related to the number of doses of benzodiazepine $(p=0.066)$; children having prehospital treatment were more likely to receive more than 2 doses $(p=0.001)$, and those with prehospital treatment were more likely to have respiratory depression $(\mathrm{p}=0.01)$. The mean duration of ventilatory support was 15 hours and was independent of etiology $(p=0.16)$. Five $(5 \%)$ children with SE died; 2 with meningitis, 1 each with hepatic failure, brain tumor, and neurodegenerative disease. Appropriate pre-PIC treatment of SE is stressed to avoid the necessity for 2 doses of benzodiazepines and the increased risk of respiratory depression. (Chin RFM, Verhulst I, Neville BGR, et al. Inappropriate emergency management of status epilepticus in children contributes to need for intensive care. $\mathbf{J}$ Neurol Neurosurg Psychiatry November 2004;75:1584-1588). (Respond: Dr RFM Chin, Neurosciences Unit, Institute of Child Health, University College and Great Ormond Street Hospital for Children, London WC1N 1EH, UK).

COMMENT. The risks of morbidity and mortality of status epilepticus are adversely affected by delayed and inadequate treatment. Information regarding the prehospital treatment of SE in individual patients is essential to avoid administration of excess benzodiazepines and an increased risk of respiratory depression. Improved prehospital management of SE may require revision of guidelines and the use of alternative medications administered by routes more acceptable than rectal. The use of intravenous agents is limited by the difficulty of administration in young children treated in an outpatient clinic. Recent studies have demonstrated similar efficacy to rectal diazepam in controlled trials of buccal and nasal preparations (Scott RC et al. Lancet 1999;353:623-626; O'Regan ME et al. Dev Med Child Neurol 1996;38:1037-1045). 